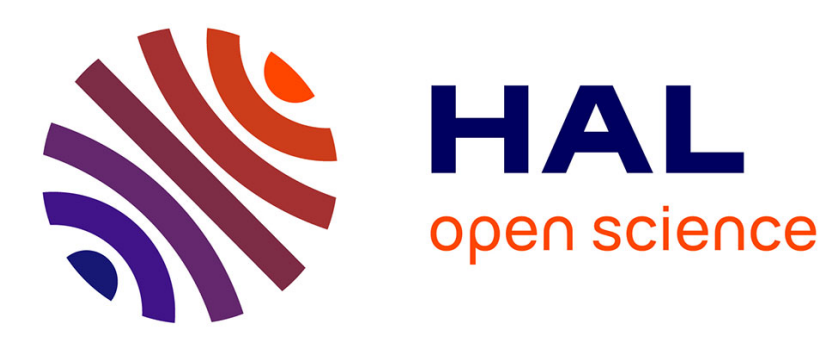

\title{
Thermal fluctuations of a liquid film on a heterogeneous solid substrate
}

Olivier Pierre-Louis

\section{To cite this version:}

Olivier Pierre-Louis. Thermal fluctuations of a liquid film on a heterogeneous solid substrate. Physical Review E , 2016, 94, pp.032802. 10.1103/PhysRevE.94.032802 . hal-02290212

\section{HAL Id: hal-02290212 \\ https://univ-lyon1.hal.science/hal-02290212}

Submitted on 16 Feb 2021

HAL is a multi-disciplinary open access archive for the deposit and dissemination of scientific research documents, whether they are published or not. The documents may come from teaching and research institutions in France or abroad, or from public or private research centers.
L'archive ouverte pluridisciplinaire HAL, est destinée au dépôt et à la diffusion de documents scientifiques de niveau recherche, publiés ou non, émanant des établissements d'enseignement et de recherche français ou étrangers, des laboratoires publics ou privés. 


\title{
Thermal fluctuations of a liquid film on a heterogeneous solid substrate
}

\author{
Olivier Pierre-Louis \\ Institut Lumière Matière, UMR5306 Université Lyon 1-CNRS, Université de Lyon, 69622 Villeurbanne, France
}

(Received 5 July 2016; published 29 September 2016)

\begin{abstract}
The dynamics of the fluctuations of a liquid film on a heterogeneous substrate is analyzed. We consider the case of a viscous liquid in the Stokes limit, with small variations of substrate height and a small varying slip length. We discuss the possibility of extracting the topographic profile or the slip length profile at the liquid-solid interface from the measurement of the fluctuations of the free liquid surface. Our results, therefore, explore the theoretical basis of a strategy for a fluctuation-induced microscopy of immersed solids at the micrometer scale.
\end{abstract}

DOI: 10.1103/PhysRevE.94.032802

\section{INTRODUCTION}

Probing heterogeneities inside a liquid or solid bulk using the measurement of waves or fluctuations at the surface is an old idea. For example, one of the most powerful techniques to probe the deep structure of the earth is to measure seismic waves at its surface [1]. Indeed, waves carry information about the media that they have crossed, or the obstacles that they have met. Such an approach enters into the category of the so-called inverse problems, which has been the subject of a very large literature, in physics, mathematics, and engineering. In this work, we propose to probe the heterogeneities of a substrate covered by a liquid film from the measurement of the timecorrelation function of the free-surface thermal fluctuations.

This study is motivated by two recent experimental advances in the measurement of liquid surface thermal fluctuations. The first one is based on the direct optical observation of the interface of phase-separated colloid-polymer dispersions [2], which exhibits micrometer-scale roughnesses, and capillary times of the order of seconds. The second one is the surface fluctuation specular refection (SFSR) [3], which allows one to probe the local time-dependent fluctuations of the free surface of usual molecular liquids despite the small surface roughness of the order of angstroms, using the deviation of a specular laser beam. The SFSR technique allows one to probe free-surface fluctuations on spatial lateral scales ranging from a few micrometers to a few hundred micrometers, and on time scales between $1 \mathrm{~s}$ and $10 \mu \mathrm{s}$ [3]. Providing local measurements of surface fluctuations, these two techniques allow one to measure the spatial variations of the fluctuations. Here, we show how these spatial variations contain information about surface heterogeneities, such as topographical patterns, or changes in the local slip length at the liquid-solid interface.

Our results, therefore, lead to alternative perspectives in the visualization of solids immersed in liquids. Indeed, visualizing substrate heterogeneities from the measurement of surface fluctuations has at least two advantages. First, it is a noninvasive method that does not perturb the system. This is a crucial feature for measurements in soft matter and biological systems, which are often easily altered by small perturbations. Second, in contrast to optical visualization techniques, fluctuation-mediated methods allow one to study substrates under opaque liquids, or invisible interfaces in transparent index-matched liquid-substrate systems.

To be more specific, we consider a film of viscous liquid in the Stokes regime, i.e., when inertial effects are negligible.
We assume that the free surface is far enough from the liquid-substrate interface so that the equilibrium shape and static equilibrium fluctuations do not depend on the substrate profile. As discussed below, this regime is usually reached for film thicknesses larger than a micrometer. We also assume that the substrate height variations and the slip length are small compared to the film depth. Indeed, the slip length is usually of the order of nanometers, or tens of nanometers [4], and it can be increased up to micrometers using superhydrophobic surfaces [5]. Within these assumptions, we analyze the effect of substrate profile perturbations and of inhomogeneities in the interfacial slip length on the dynamics of free-surface fluctuations.

We consider both the possibility of direct observation of the space-dependent surface correlation function and the observation of space-dependent surface fluctuations via SFSR. In these two cases, we find that one can extract the substrate profile or the substrate slip length variations from the measurement of the spatial variations of the time correlation function of free-surface fluctuations. Finally, we discuss recent experiments [6], and we present some possible directions for future work.

\section{MODEL EQUATIONS}

We start with the presentation of the model equations. Consider a liquid film limited by a free liquid-gas surface at $z=h(x, y, t)$, and a substrate at $z=h_{s}(x, y)$. A schematic of the system is shown in Fig. 1.

We neglect the viscosity of the gas above the liquid, and we assume that the substrate is perfectly rigid. The fluid velocity $\mathbf{u}(x, y, z, t)$ obeys

$$
\eta \nabla^{2} \mathbf{u}=\nabla p
$$

where $\eta$ is the dynamic viscosity and $p(x, y, z, t)$ is the pressure. This corresponds to the Stokes regime, which models a liquid at small Reynolds numbers, where the convective term $\sim \mathbf{u}$. $\nabla \mathbf{u}$ and the acceleration term $\sim \partial_{t} \mathbf{u}$ can be neglected from the full Navier-Stokes equation. This regime is usually valid in the case of colloidal systems, as shown in Ref. [2]. Moreover, as discussed in Ref. [3], inertial effects for thick liquid films in SFSR are negligible in the frequency range $\omega \ll \omega_{1}$, where $\omega_{1}=\eta \rho R^{2}$, with $R$ the beam radius. For water, $\omega_{1} / 2 \pi \approx$ $200 \mathrm{~Hz}$, and for viscous oils, $\omega_{1}$ is much larger [3]. 


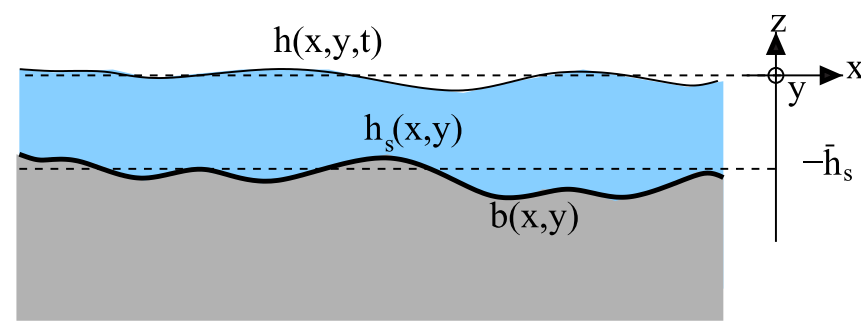

FIG. 1. Schematic of a liquid film undergoing surface fluctuations $h(x, y, t)$ over a heterogeneous substrate with height $h_{s}(x, y)$ and local slip length $b(x, y)$. that

In addition, we assume that the fluid is incompressible, so

$$
\nabla \cdot \mathbf{u}=\mathbf{0} .
$$

The fluid is in contact with a nonflat substrate of height $h_{s}(x, y)$ along $z$, with a spatially varying friction coefficient $\beta(x, y)$, as depicted in Fig. 1. The boundary condition at the substrate surface $z=h_{s}(x, y)$ then reads

$$
\left(\hat{\mathbf{n}} \cdot \sigma^{\prime}\right)_{\|}=\beta(x, y) \mathbf{u}_{\|},
$$

where for any vector field a we define the parallel component to the surface as $\mathbf{a} \|=\mathbf{a}-\hat{\mathbf{n}}_{s}\left(\hat{\mathbf{n}}_{s} \cdot \mathbf{a}\right)$, with $\hat{\mathbf{n}}_{s}$ a unit vector normal to the substrate. In addition, the components of the viscous stress tensor $\sigma^{\prime}$ are

$$
\sigma_{i j}^{\prime}=\eta\left(\partial_{i} u_{j}+\partial_{j} u_{i}\right) .
$$

At the liquid-gas interface $z=h(x, y, t)$, mechanical equilibrium imposes (see Ref. [7], p. 342)

$$
\sigma \cdot \hat{\mathbf{n}}=-(\Pi+\gamma \kappa) \hat{\mathbf{n}},
$$

where $\hat{\mathbf{n}}$ is the outward normal to the free surface, $\kappa$ is the mean curvature, $\gamma$ is the surface tension, and

$$
\sigma_{i j}=\eta\left(\partial_{i} u_{j}+\partial_{j} u_{i}\right)-\delta_{i j} p=\sigma_{i j}^{\prime}-\delta_{i j} p
$$

are the component of the liquid stress tensor. Moreover, $\Pi$ is the disjoining pressure, which results from the free-energy variation when displacing the free-surface position $h$.

Finally, we write down the kinematic law obeyed by the free surface,

$$
\partial_{t} h(x, y, t)=u_{z}(x, y, h, t)-\mathbf{u}_{\{x, y\}}(x, y, h, t) \cdot \nabla_{\{x, y\}} h,
$$

where $\{x, y\}$ indicates $2 \mathrm{D}$ vectors and operators in the $x, y$ plane.

\section{RELAXATION RATE OF FREE-SURFACE FLUCTUATIONS}

We now wish to determine the spatial dependence of the relaxation rate of free-surface fluctuations in the presence of a heterogeneous substrate. An important assumption here is that the equilibrium (i.e., static, or average) profile of the free surface is assumed to be flat. This requires that the free surface is far enough from the substrate for its equilibrium profile to be unaffected by the shape or properties of the substrate, i.e., $\Pi$ does not depend on $h_{s}$. A quantitative discussion of this approximation, which is provided in Appendix A, indicates that this assumption is valid for film with thicknesses of at least some micrometers, and away from criticality where large fluctuations of the free surface can induce entropic interactions with the substrate.

Let us assume a small free-surface perturbation $h(x, y, t)=$ $\bar{h}+\delta h(x, y, t)$. In the following, we set the origin of the $z$ axis such that $\bar{h}=0$. We also consider small perturbations of a flat substrate,

$$
h_{s}(x, y)=-\bar{h}_{s}+h_{s}^{(1)}(x, y) \text {, }
$$

and small slip lengths,

$$
b(x, y)=\eta / \beta(x, y) .
$$

Defining

$$
\delta h_{s}(x, y)=h_{s}^{(1)}(x, y)+b(x, y),
$$

we focus on the limit $\delta h \ll \delta h_{s} \ll \bar{h}_{s}$.

The details of the derivation of the surface relaxation rate starting from the model equations presented in the previous section are reported in Appendix B. The results are summarized in the dispersion relation

$$
i \omega \delta h_{\omega \mathbf{q}}=\int d \mathbf{q}^{\prime} \alpha_{\mathbf{q q}^{\prime}} \epsilon_{\mathbf{q}^{\prime}} \delta h_{\omega \mathbf{q}^{\prime}}
$$

where the Fourier transform of a function $f(x, y, t)$ in the $x$ and $y$ directions and in time is defined as

$$
f_{\omega \mathbf{q}}=\int d t \int d x \int d y f(x, y, t) e^{-i\left(q_{x} x+q_{y} y\right)-i \omega t},
$$

with $\mathbf{q}=\left(q_{x}, q_{y}\right)$. In addition, we have defined

$$
\begin{gathered}
\epsilon_{q}=\Pi^{\prime}(\bar{h})+\gamma q^{2} . \\
\alpha_{\mathbf{q q}^{\prime}}=\tilde{\alpha}_{\mathbf{q}}^{(0)} \delta_{\mathbf{q}-\mathbf{q}^{\prime}}+\alpha_{\mathbf{q q}^{\prime}}^{(1)},
\end{gathered}
$$

with

$$
\begin{gathered}
\tilde{\alpha}_{\mathbf{q}}^{(0)}=-\frac{1}{2 \eta q} \frac{\cosh q \bar{h}_{s} \sinh q \bar{h}_{s}-q \bar{h}_{s}}{\cosh ^{2} q \bar{h}_{s}+\left(q \bar{h}_{s}\right)^{2}}, \\
\alpha_{\mathbf{q q}^{\prime}}^{(1)}=\frac{1}{(2 \pi)^{2} \eta} \delta h_{s \mathbf{q}-\mathbf{q}^{\prime}}\left(\mathbf{K}_{\mathbf{q}} \cdot \mathbf{K}_{\mathbf{q}^{\prime}}\right), \\
\mathbf{K}_{\mathbf{q}}=\frac{\mathbf{q} \bar{h}_{s} \cosh q \bar{h}_{s}}{\cosh ^{2} q \bar{h}_{s}+\left(q \bar{h}_{s}\right)^{2}},
\end{gathered}
$$

where $q=|\mathbf{q}|$.

The expression of $\tilde{\alpha}_{\mathbf{q}}^{(0)}$ is in agreement with the dispersion relation given in Refs. [8,9] for a flat substrate:

$$
i \omega^{(0)}=\tilde{\alpha}_{\mathbf{q}}^{(0)} \epsilon_{\mathbf{q}} \equiv \mu_{q}
$$

The main focus of this paper is to analyze the consequences of the first-order term $\alpha_{\mathbf{q q}^{\prime}}^{(1)}$ caused by surface heterogeneities on surface fluctuation dynamics. 


\section{THERMAL FLUCTUATIONS}

\section{A. Langevin formalism}

To account for thermal fluctuations, we add a Langevin force to Eq. (11), leading to

$$
i \omega \delta h_{\omega \mathbf{q}}=\int d \mathbf{q}^{\prime} \alpha_{\mathbf{q q}^{\prime}} \epsilon_{\mathbf{q}^{\prime}} \delta h_{\omega \mathbf{q}^{\prime}}+\frac{1}{\eta} \mathcal{N}_{\omega \mathbf{q}}
$$

We need to derive the correlations of the Langevin force $\mathcal{N}_{\omega \mathbf{q}}$. A derivation of the noise correlations can be obtained using fluctuating hydrodynamics $[7,10,11]$. However, since we are interested in small linear perturbations $\delta h$ of the free-surface profile at thermodynamic equilibrium, a shorter derivation based on the fluctuation dissipation theorem is possible.

Indeed, at thermodynamic equilibrium, the static spectrum is fixed by equipartition. Since the disjoining pressure $\Pi$ is taken to be independent of the substrate profile $h_{s}(x, y)$, the system free-energy density $\gamma(\nabla \delta h)^{2} / 2+\Pi^{\prime}(\bar{h}) \delta h^{2} / 2$ is invariant under translations of $\delta h$ in the $x, y$ plane. As a consequence, equipartition takes the same form as in a spatially homogeneous system,

$$
\left\langle\left|\delta h_{\mathbf{q}}\right|^{2}\right\rangle_{\text {stat }}=\frac{k_{B} T}{\epsilon_{q}},
$$

where we have defined the static spectrum as

$$
\left\langle\left|\delta h_{\mathbf{q}_{1}}\right|^{2}\right\rangle_{\text {stat }}=\int \frac{d \mathbf{q}_{2}}{(2 \pi)^{2}} \int \frac{d \omega_{1}}{2 \pi} \int \frac{d \omega_{2}}{2 \pi}\left\langle\delta h_{\omega_{1} \mathbf{q}_{1}} \delta h_{\omega_{2} \mathbf{q}_{2}}\right\rangle .
$$

Imposing Eq. (20) in Eq. (19), we obtain the correlation of the noise,

$$
\left\langle\mathcal{N}_{\omega_{1} \mathbf{q}_{1}} \mathcal{N}_{\omega_{2} \mathbf{q}_{2}}\right\rangle=-2 \pi \delta_{\omega_{1}+\omega_{2}} 2 k_{B} T \eta^{2}(2 \pi)^{2} \alpha_{\mathbf{q}_{1}-\mathbf{q}_{2}} .
$$

\section{B. Two-point time correlation function}

Combining Eq. (22) in Eq. (19), one may calculate the two-point correlation function of the free-surface fluctuations:

$$
\left\langle\delta h_{\omega_{1} \mathbf{q}_{1}} \delta h_{\omega_{2} \mathbf{q}_{2}}\right\rangle=2 \pi \delta_{\omega_{1}+\omega_{2}} G_{\omega_{1} \mathbf{q}_{1} \mathbf{q}_{2}}
$$

with

$$
\begin{aligned}
G_{\omega_{1} \mathbf{q}_{1} \mathbf{q}_{2}}= & 2 k_{B} T(2 \pi)^{2}\left[\delta_{\mathbf{q}_{1}+\mathbf{q}_{2}} \frac{-\tilde{\alpha}_{\mathbf{q}_{1}}^{(0)}}{\omega_{1}^{2}+\mu_{q_{1}}^{2}}\right. \\
& \left.-\alpha_{\mathbf{q}_{1}-\mathbf{q}_{2}}^{(1)} \frac{\omega_{1}^{2}-\mu_{q_{1}} \mu_{q_{2}}}{\left(\omega_{1}^{2}+\mu_{q_{1}}^{2}\right)\left(\omega_{1}^{2}+\mu_{q_{2}}^{2}\right)}\right] .
\end{aligned}
$$

Since it is constrained by translational invariance in time, the two-point dynamic correlation function can be defined as

$$
s\left(\mathbf{r}_{1}, \mathbf{r}_{2}, t\right)=\left\langle\delta h\left(\mathbf{r}_{1}, t_{0}\right) \delta h\left(\mathbf{r}_{2}, t_{0}+t\right)\right\rangle .
$$

Using Eq. (23), we find a simple exponential relaxation for the unperturbed substrate [i.e., flat and with no slip, with $\left.h_{s}(x, y)=-\bar{h}_{s}\right]$ :

$$
s_{\mathbf{q}_{1} \mathbf{q}_{2}}^{(0)}(t)=(2 \pi)^{2} \delta_{\mathbf{q}_{1}+\mathbf{q}_{2}}\left\langle\left|\delta h_{\mathbf{q}_{1}}\right|^{2}\right\rangle_{\text {stat }} e^{-\mu_{q_{1}}|t|}
$$

leading to

$$
s^{(0)}\left(\mathbf{r}_{1}, \mathbf{r}_{2}, t\right)=\left\langle\left|\delta h_{\mathbf{q}_{1}}\right|^{2}\right\rangle_{\text {stat }} \int \frac{d \mathbf{q}}{(2 \pi)^{2}} e^{i \mathbf{q} \cdot\left(\mathbf{r}_{1}-\mathbf{r}_{2}\right)-\mu_{q}|t|} .
$$

To first order in $\delta h_{s}$, we obtain

$$
s_{\mathbf{q}_{1} \mathbf{q}_{2}}^{(1)}(t)=-2 k_{B} T(2 \pi)^{2} \alpha_{\mathbf{q}_{1}-\mathbf{q}_{2}}^{(1)} \frac{e^{-\mu_{q_{1}}|t|}-e^{-\mu_{q_{2}}|t|}}{2\left(\mu_{q_{1}}-\mu_{q_{2}}\right)}
$$

from which $s^{(1)}\left(\mathbf{r}_{1}, \mathbf{r}_{2}, t\right)$ can be obtained once again by Fourier transform.

As a remark, since the disjoining pressure $\Pi$ is taken to be independent of $h_{s}$, the energetic term $\epsilon_{\mathbf{q}}$ is not affected by the perturbations $h_{s}^{(1)}$ of the surface profile. Thus, there is no first-order correction to $\epsilon_{\mathbf{q}}$, and no first-order correction to the static spectrum Eq. (20). The first-order correction of $s\left(\mathbf{r}_{1}, \mathbf{r}_{2}, t\right)$ should therefore vanish in the static limit $t \rightarrow 0$, and one can indeed check from Eq. (28) that as $t \rightarrow 0$,

$$
s_{\mathbf{q}_{1} \mathbf{q}_{2}}^{(1)}(t) \approx 2 k_{B} T(2 \pi)^{2} \alpha_{\mathbf{q}_{1}-\mathbf{q}_{2}}^{(1)}|t| \rightarrow 0 .
$$

Hence, the spatial heterogeneities of the signal vanish in the static limit, and the substrate profile $\delta h_{s}$ cannot be probed using the static spectrum. As a consequence, information on $\delta h_{s}$ can only be obtained from the time dependence of the fluctuations.

\section{Time autocorrelation function and SFSR}

A usual quantity analyzed in experiments, the time autocorrelation function of $\delta h(\mathbf{r}, t)$, is defined as

$$
\mathcal{S}(\mathbf{r}, t)=s(\mathbf{r}, \mathbf{r}, t)=\left\langle\delta h\left(\mathbf{r}, t_{0}\right) \delta h\left(\mathbf{r}, t_{0}+t\right)\right\rangle,
$$

where $\mathbf{r}=(x, y)$. The time-Fourier transform of this correlation function reads

$$
\mathcal{S}_{\omega}(\mathbf{r})=\int \frac{d \mathbf{q}_{1}}{(2 \pi)^{2}} \int \frac{d \mathbf{q}_{2}}{(2 \pi)^{2}} G_{\omega \mathbf{q}_{1} \mathbf{q}_{2}} e^{i\left(\mathbf{q}_{1}+\mathbf{q}_{2}\right) \cdot \mathbf{r}} .
$$

Some experiments, such as those of Ref. [2] based on the optical observation of the interface of phase-separated colloid-polymer dispersions, allow one to measure directly the time-dependent position of the free surface with optical methods. Hence, it should be possible to extract $\mathcal{S}_{\omega}(\mathbf{r})$ in this experimental system.

However, usual molecular liquids exhibit much smaller thermal roughnesses, and other types of measurements should be used. One possibility to extract information about the surface fluctuation dynamics is surface fluctuation specular reflection (SFSR) spectroscopy [3]. In this method, a laser beam is reflected specularly by the surface of a liquid. The beam is collected on two half-screens, with intensities $I_{+}$and $I_{-}$, respectively. The output of the experiments is the relative intensity

$$
\delta I=\frac{I_{+}-I_{-}}{I_{+}+I_{-}}=\int \frac{d \mathbf{q}}{(2 \pi)^{2}} h_{\mathbf{q}} F_{\mathbf{q}},
$$

with [3]

$$
F_{\mathbf{q}}=\frac{8 i \pi}{\lambda} e^{-q^{2} R^{2} / 4} \operatorname{erf}\left(\frac{q R}{2} \cos \left(\phi_{m}-\alpha\right)\right),
$$

where $\phi_{m}$ and $\alpha$ are the angles of the boundary between the two screens and of $\mathbf{q}$, respectively. Using Eq. (23), we find the 
measured normalized power spectrum:

$$
\begin{aligned}
\mathcal{S}_{\omega}(\mathbf{r}) & =\int d t e^{-i \omega t}\left\langle\delta I\left(t_{0}\right) \delta I\left(t_{0}+t\right)\right\rangle_{t_{0}} \\
& =\int \frac{d \mathbf{q}_{1}}{(2 \pi)^{2}} \int \frac{d \mathbf{q}_{2}}{(2 \pi)^{2}} F_{\mathbf{q}_{1}} F_{\mathbf{q}_{2}} G_{\omega \mathbf{q}_{1} \mathbf{q}_{2}} e^{i\left(\mathbf{q}_{1}+\mathbf{q}_{2}\right) \cdot \mathbf{r}} .
\end{aligned}
$$

Here we have used the same notation $\mathcal{S}_{\omega}(\mathbf{r})$ for the result of SFSR in Eq. (34), and for the local time-correlation function in Eq. (31). Indeed, the expressions are very similar. The only difference is the presence of $F_{q}$ in SFSR, which can be formally taken to be equal to 1 in the case of the local time-correlation function. In the following, we use the same notation $\mathcal{S}_{\omega}(\mathbf{r})$ to discuss both cases simultaneously.

As an additional remark, since the time scales are controlled by the viscosity of the liquid, we notice from Eqs. (11), (15), and (16) that the relaxation times are proportional to $\eta$. As a consequence, using Eq. (24) and Eqs. (31) and (34), the spectrum $\mathcal{S}_{\omega}$ on $\eta$ is seen to obey a simple scaling relation,

$$
\mathcal{S}_{\omega}=\eta f(\eta \omega)
$$

where the function $f(x)$ is independent of $\eta$.

From Eq. (34), combined with Eq. (24), we find the power spectrum of a flat substrate with no slip, i.e., $\delta h_{s}=0$ :

$$
\mathcal{S}_{\omega}^{(0)}=2 k_{B} T \int \frac{d \mathbf{q}_{1}}{(2 \pi)^{2}} F_{\mathbf{q}_{1}} F_{-\mathbf{q}_{1}} \frac{-\tilde{\alpha}_{\mathbf{q}_{1}}^{(0)}}{\omega_{1}^{2}+\mu_{q_{1}}^{2}} .
$$

The spectrum $\mathcal{S}_{\omega}^{(0)}$, plotted in Figs. 2(a) and 2(b) and Figs. 3(a) and 3(b), has been obtained experimentally in SFSR [6]. The observed spectrum was in quantitative agreement with Eq. (36), showing the effect of the finite depth of the film on surface fluctuation dynamics.

In general, the scaling form Eq. (35) imposes that the decrease of $\eta$ by some factor leads to a simple shift of $\mathcal{S}_{\omega}^{(0)}$ down and to the right in log-log scale. For example, in the specific case of direct measurement $\left(F_{\mathbf{q}}=1\right)$, the function $f(x)$ that intervenes in Eq. (35) is $\sim 1 / x$ when $x$ is large, leading to $\mathcal{S}_{\omega}^{(0)} \sim 1 / \omega$ independent of $\eta$ for large $\omega$. In contrast, $f(x) \sim 1 / x^{2}$ for SFSR, leading to $\mathcal{S}_{\omega}^{(0)} \sim 1 /\left(\eta \omega^{2}\right)$. Let us be more precise: in the limit of large frequencies $\omega \rightarrow \infty$, one has for direct measurement

$$
\mathcal{S}_{\omega}^{(0)} \approx \frac{k_{B} T}{8 \gamma \omega} .
$$

In the case of SFSR,

$$
\mathcal{S}_{\omega}^{(0)} \approx v\left(\bar{h}_{s} / R\right) \frac{k_{B} T}{\lambda^{2} \eta \omega^{2}},
$$

where $v(x)$ is a function of $x$ obeying $v(x) \rightarrow 14.9 \ldots$ when $x \gg 1$, and $v(x) \rightarrow x^{3} \times 49.1 \ldots$ when $x \ll 1$.

In the opposite limit of low frequencies, the spectrum is dominated by the long-wavelength lubrication behavior. For $\omega \rightarrow 0$, we find for direct measurement

$$
\mathcal{S}_{\omega}^{(0)} \approx \frac{k_{B} T}{2 \gamma \omega},
$$
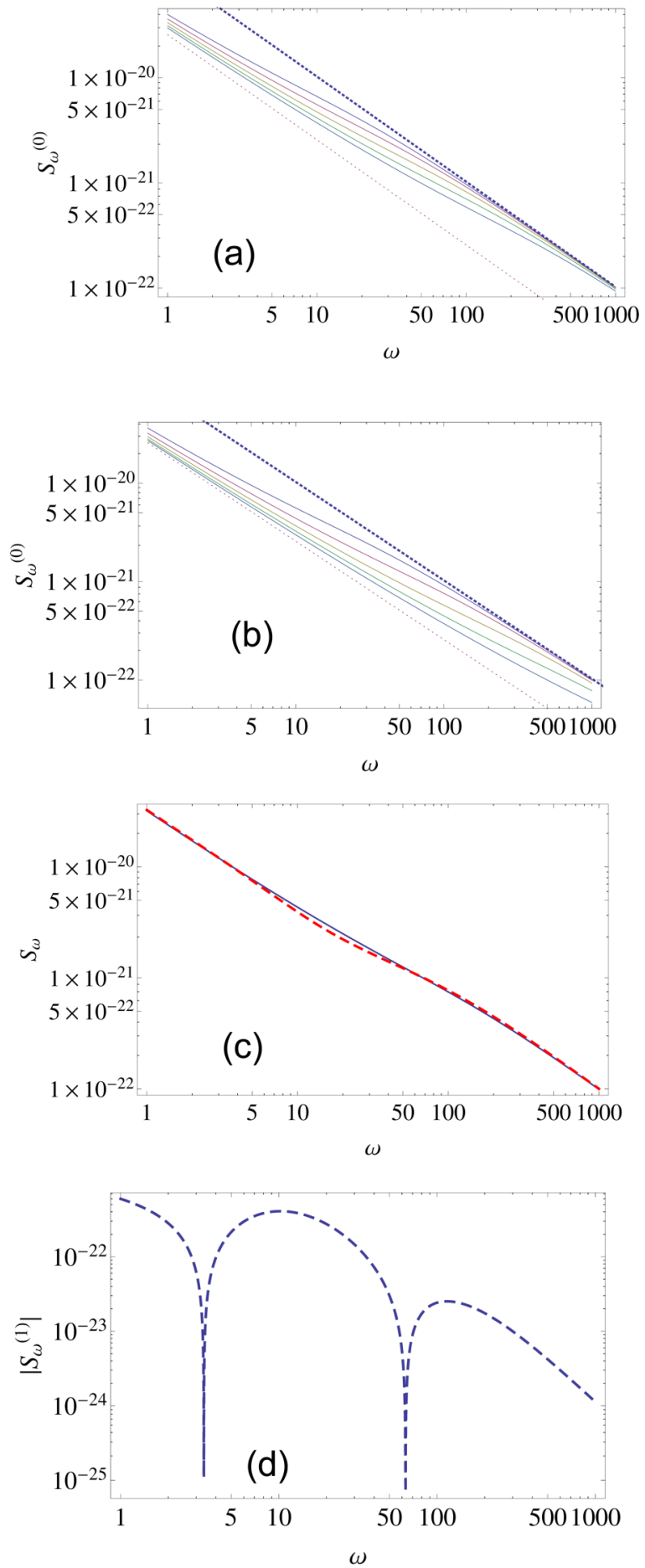

FIG. 2. Time autocorrelation power spectrum $\mathcal{S}_{\omega}$ as a function of $\omega$ (in s ${ }^{-1}$ ). Parameters: $\gamma=20 \times 10^{-3} \mathrm{~J} \mathrm{~m}^{-2}, T=298 \mathrm{~K}$. The dotted and thick-dotted lines are, respectively, the high-frequency [Eq. (37)] and low-frequency [Eq. (39)] limits. (a) Flat substrate with $\eta=3.9$ Pa s, and various depths $\bar{h}_{s}=\infty$ and $\bar{h}_{s}=2^{-n} \times 500 \mu \mathrm{m}$, with $n=$ $0,1,2,3,4$. (b) Flat substrate with $\bar{h}_{s}=100 \mu \mathrm{m}$ and $\eta=3^{-n}$ Pa s with $n=0, \ldots, 4$. (c) Substrate with parallel grooves of a sinusoidal cross section, with $\bar{h}_{s}=80 \mu \mathrm{m}, \delta h_{s}=50 \mu \mathrm{m}, \lambda=250 \mu \mathrm{m}$, and $\eta=3.9$ $\mathrm{Pa}$. The dashed (red) line is the spectrum calculated over the maxima of the pattern. The solid (black) line represents the spectrum of the flat substrate. (d) Absolute value of the first-order contribution $\left|\mathcal{S}_{\omega}^{(1)}\right|$, with the same parameters as in (c). 

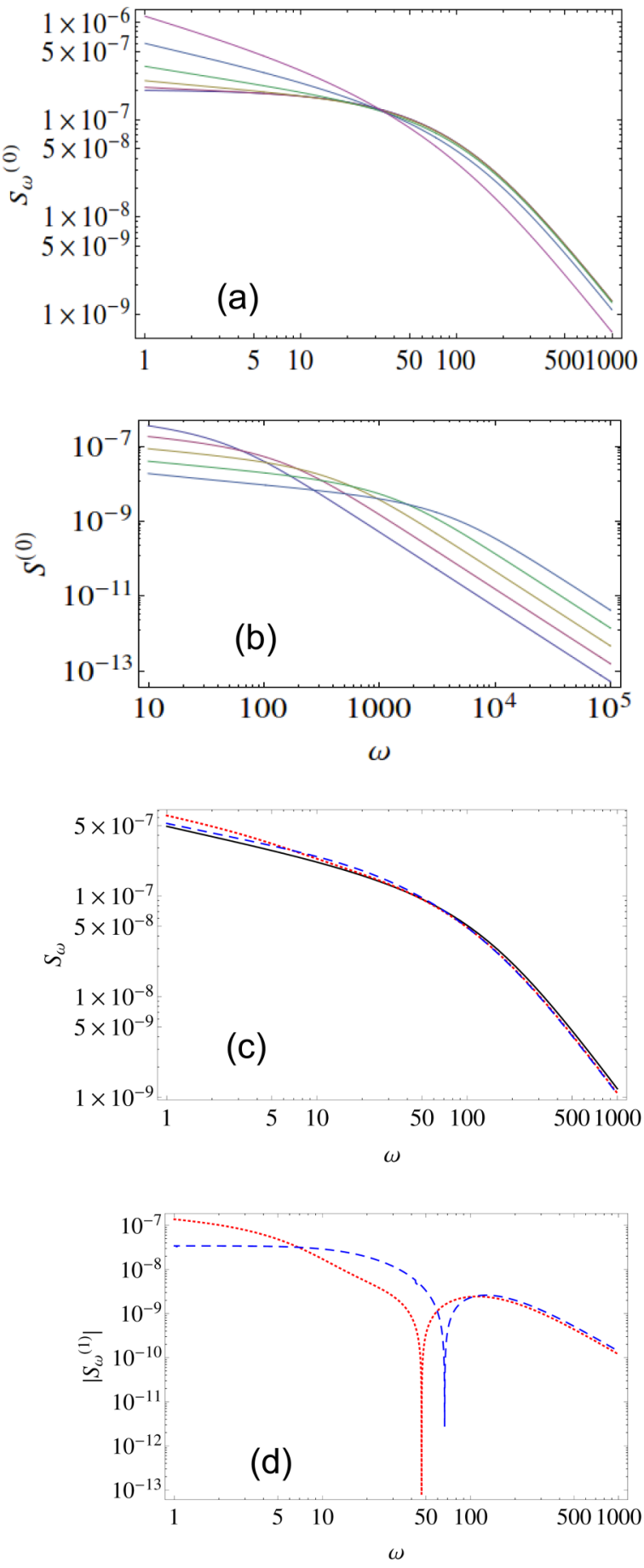

FIG. 3. SFSR spectrum $\mathcal{S}_{\omega}$ as a function of $\omega\left(\right.$ in s $\left.^{-1}\right)$. Parameters: $\gamma=20 \times 10^{-3} \mathrm{~J} \mathrm{~m}^{-2}, R=28 \mu \mathrm{m}, T=298 \mathrm{~K}$, and $\lambda=632.8 \mathrm{~nm}$. (a) Flat substrate with $\eta=3.9 \mathrm{~Pa} \mathrm{~s}$, and various depths $\bar{h}_{s}=2^{-n} \times$ $500 \mu \mathrm{m}$, with $n=0, \ldots, 5$. The thick-dotted line corresponds to the limit $\bar{h}_{s}=\infty$, and the dotted line corresponds to the low-frequency limit Eq. (40) for $n=5$. (b) Flat substrate with $\bar{h}_{s}=100 \mu \mathrm{m}$ and $\eta=3^{-n}$ Pa s with $n=0, \ldots, 4$. (c) Substrate with parallel grooves of a sinusoidal cross section, with $\bar{h}_{s}=80 \mu \mathrm{m}, \delta h_{s}=50 \mu \mathrm{m}, \lambda=$ $250 \mu \mathrm{m}$, and $\eta=3.9 \mathrm{~Pa}$ s. The dotted (red) and dashed (blue) lines are the spectra calculated over the maxima of the pattern for $\phi_{m}=0$ and $\pi / 2$, respectively. The solid (black) line represents the spectrum of the flat substrate. (d) Absolute value of the first-order contribution $\left|\mathcal{S}_{\omega}^{(1)}\right|$, with the same parameters as in (c).

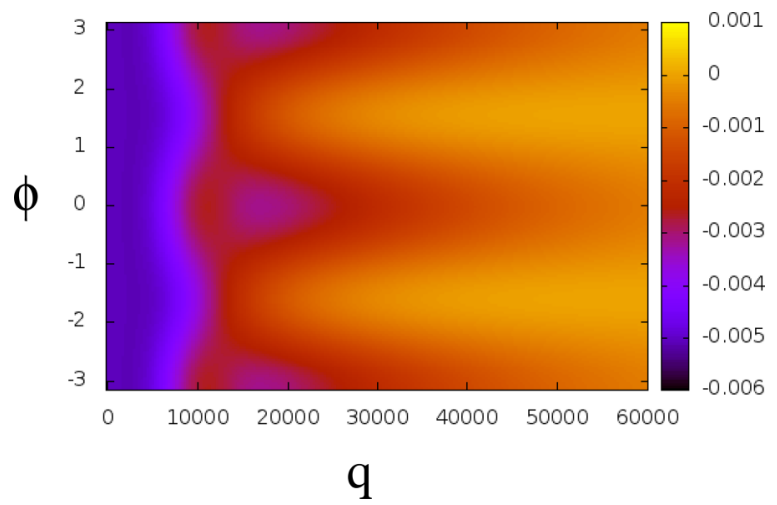

FIG. 4. Plot of $\mathcal{C}_{\omega \mathbf{q}}^{-1}$ in the SFSR case, with $\mathbf{q}=q(\cos \phi, \sin \phi)$ (in $\mathrm{m}^{-1}$ ). Numerical parameters are similar to those of Ref. [6]: $\gamma=$ $20 \times 10^{-3} \mathrm{~J} \mathrm{~m}^{-2}, \bar{h}_{s}=80 \mu \mathrm{m}, \eta=3.9 \mathrm{~Pa} \mathrm{~s}, R=28 \mu \mathrm{m}, T=298 \mathrm{~K}$, and $\lambda=632.8 \mathrm{~nm}$.

and for SFSR,

$$
\mathcal{S}_{\omega}^{(0)} \approx \frac{4 \pi 6^{1 / 2} \eta^{1 / 2} k_{B} T R^{2}}{\lambda^{2} \gamma^{3 / 2} \bar{h}_{s}^{3 / 2} \omega^{1 / 2}} .
$$

Plots of $\mathcal{S}_{\omega}^{(0)}$, together with the low- and high-frequency limits, are shown in Figs. 2(a) and 2(b) and Figs. 3(a) and 3(b).

From Eq. (34), combined with Eqs. (24) and (16), we find to first order

$$
\mathcal{S}_{\omega \mathbf{q}}^{(1)}=\delta h_{s \mathbf{q}} \mathcal{C}_{\omega \mathbf{q}}^{-1},
$$

where

$$
\begin{aligned}
\mathcal{C}_{\omega \mathbf{q}}^{-1}= & -\frac{2 k_{B} T}{\eta} \int \frac{d \mathbf{q}_{1}}{(2 \pi)^{2}} F_{\mathbf{q}_{1}} F_{\mathbf{q}-\mathbf{q}_{1}}\left(\mathbf{K}_{\mathbf{q}_{1}} \cdot \mathbf{K}_{\mathbf{q}-\mathbf{q}_{1}}\right) \\
& \times \frac{\omega^{2}-\mu_{q_{1}} \mu_{\left|\mathbf{q}-\mathbf{q}_{1}\right|}}{\left(\omega^{2}+\mu_{q_{1}}^{2}\right)\left(\omega^{2}+\mu_{\left|\mathbf{q}-\mathbf{q}_{1}\right|}^{2}\right)} .
\end{aligned}
$$

An example of $\mathcal{C}_{\omega \mathbf{q}}^{-1}$ is shown in Fig. 4 for the SFSR case, with parameters similar to those of Ref. [6]. The first-order contribution $\mathcal{S}_{\omega \mathbf{q}}^{(1)}$ also obeys some scaling relation at high frequencies $\omega \rightarrow \infty$, namely $\mathcal{S}_{\omega \mathbf{q}}^{(1)} \sim 1 /\left(\eta \omega^{2}\right)$ both for direct measurement and for SFSR.

As an example, the spectrum above a sinusoidal surface is plotted in Figs. 2(c) and 2(d) and Figs. 3(c) and 3(d). Other surface profiles, with square patterns or a Gaussian protrusion, are discussed in Appendix C.

As already discussed above, the static spectrum of $\left\langle\left|h_{q}\right|^{2}\right\rangle_{\text {stat }}$ is fixed by equipartition, and the substrate profile corrections have no effect on the static spectrum. As a consequence, $\int(d \omega / 2 \pi) \mathcal{S}_{\omega}^{(1)}=0$, and

$$
\int \frac{d \omega}{2 \pi} \mathcal{C}_{\omega \mathbf{q}}^{-1}=0 .
$$

This is mathematically rooted in the identity for $a>0$, and $b>0$ :

$$
\int \frac{d \omega}{2 \pi} \frac{\omega^{2}-a b}{\left(\omega^{2}+a^{2}\right)\left(\omega^{2}+b^{2}\right)}=0 .
$$

The relation Eq. (43) shows that $\mathcal{S}_{\omega}^{(1)}$ should change sign when $\omega$ varies. This is clearly seen in Figs. 2(c) and 2(d) and Figs. 3(c) and 3(d). 


\section{EXTRACTING THE SUBSTRATE PROFILE}

In direct space, the spatial variations of $\mathcal{S}_{\omega}^{(1)}(\mathbf{r})$ can be explicitly related to the surface profile from the Fourier transform of Eq. (41):

$$
\mathcal{S}_{\omega}^{(1)}(\mathbf{r})=\int d \mathbf{r}^{\prime} \delta h_{s}\left(\mathbf{r}^{\prime}\right) \mathcal{C}_{\omega}^{-1}\left(\mathbf{r}-\mathbf{r}^{\prime}\right),
$$

where $\mathcal{C}_{\omega}^{-1}(\mathbf{r})$ is the space Fourier transform of $\mathcal{C}_{\omega \mathbf{q}}^{-1}$. Inverting this relation, the substrate profile can be extracted from the spatial variations of the time autocorrelation spectrum or of the SFSR spectrum as

$$
\delta h_{s}(\mathbf{r})=\int d \mathbf{r}^{\prime} \mathcal{S}_{\omega}^{(1)}\left(\mathbf{r}-\mathbf{r}^{\prime}\right) \mathcal{C}_{\omega}\left(\mathbf{r}^{\prime}\right),
$$

where $\mathcal{C}_{\omega}(\mathbf{r})$ is defined via its Fourier transform $\mathcal{C}_{\omega \mathbf{q}}=1 / \mathcal{C}_{\omega \mathbf{q}}^{-1}$. We notice here that any value of $\omega$ can be used to obtain $\delta h_{s}(\mathbf{r})$.

Two important remarks are now in order. First, $\mathcal{C}_{\omega}^{-1}$ is a function of the average depth $\bar{h}_{s}$. Therefore, the average depth should be determined by some other measurement. A first possibility is that there is a region somewhere where it is known a priori that the substrate is flat and without slip. Then, this region can be taken as a reference to extract $\bar{h}_{s}$ from a fit of the time correlation function spectrum $\mathcal{S}_{\omega}^{(0)}$ of a flat substrate. In the absence of slip, a second method can be used. Indeed, from an experiment where $\mathcal{S}_{\omega}$ is measured, $\mathcal{S}_{\omega}^{(0)}$ can be obtained from spatial averaging. Then, one can extract $\bar{h}_{s}$ from $\mathcal{S}_{\omega}^{(0)}$.

As a second remark, we notice that we only obtain the quantity $\delta h_{s}=h_{s}^{(1)}+b$. The two contributions-from the substrate profile and from the slip - cannot be separated from the measurement of the correlation functions within our formalism. However, if we assume a no-slip boundary condition, we can extract the substrate profile, and if we assume a flat substrate profile, we can obtain the spatial dependence of the slip length.

\section{CONCLUSION}

The relation (46) may be interpreted as a substrate profile visualization method, or in other words, a fluctuation-mediated microscopy technique. As discussed in the Introduction, such a visualization method is interesting because it is nonintrusive. Another advantage is that one can probe the substrate-liquid interface in transparent or opaque systems, which cannot be observed with standard optical techniques. In addition, this strategy also allows one to gain information on the heterogeneity of the slip between the liquid and the substrate. However, one still needs to prove its feasibility from a given experimental setup.

A recent attempt to measure fluctuations over a patterned substrate has been reported in Ref. [6]. In this work, the pattern was made of gratings with a rectangular section (height $100 \mu \mathrm{m}$, period $250 \mu \mathrm{m}$ ), and no slip is expected. Although the pattern could be clearly detected from the spatial variations of the fluctuation spectrum, the present theory cannot be applied because the amplitude of the pattern was of the same order as the average depth to obtain a large enough signal. Interestingly, the experimental results show that the spatially averaged spectrum over one period of a periodic pattern is isotropic, but different from the spectrum of a flat substrate with an effective depth. In contrast, our linear perturbation approach predicts that all the consequences of the patterns should vanish when averaging over one period, and the averaged spectrum should be equal to that of a flat substrate with average depth. Since our linear model does not account for these experimental findings, it would be interesting to expand the substrate boundary condition Eq. (5) beyond the linear approximation, i.e., to higher orders in $\delta h_{s}$. In addition, since the functional dependence on the substrate profile and on the slip is different in the model equations [see Eq. (5)], the analysis of higher-order contributions should also allow one to distinguish slip from profile perturbations.

Another interesting perspective is to measure the heterogeneity of slip lengths in the presence of Cassie-Baxter states $[12,13]$ where a layer of gas is trapped at the substrate surface by an array of pillars (or other microstructures [14]). This perspective could be achieved with SFSR since the typical liquid film depths are of the order of several microns to tens of microns [6], and typical slip lengths can be increased up to several microns [5]. Since Cassie-Baxter states also correspond to large contact angles, thin films should easily dewet the substrates, and it is an experimental challenge to keep a stable thin liquid film in contact with a substrate in the Cassie-Baxter state. In addition, surface fluctuations of the interface between the liquid and the gas under the film would also enter into play. Nevertheless, the measurement of the variations of the surface fluctuations could provide information on the heterogeneities in the slip, while previous experimental investigations have essentially been devoted to the measurement of the average slip length [5].

Finally, an important direction is to extend the method presented here to study complex fluids. Indeed, recent experiments on the fluctuation of polymer melts on periodically patterned substrates results in a nontrivial anisotropy of the fluctuations at large scales, as measured by x-ray photon correlation spectroscopy (XPCS) [15]. More generally, patterned substrates could help to probe the dynamics of complex fluids in heterogeneous and confined environments, where both the bulk dynamics [16] and the slip condition [17,18] are nontrivial.

\section{ACKNOWLEDGMENTS}

The author thanks L. Talini and T. Bickel for fruitful discussions, and for remarks on the manuscript.

\section{APPENDIX A: ASSUMPTION OF A FLAT EQUILIBRIUM PROFILE FOR THE FREE SURFACE}

In this Appendix, the amplitude of the spatial variations of the static profile of a thin film is discussed, following the approach presented in Ref. [19]. Since surface tension kills the small-wavelength perturbations of the free surface, the most dangerous modes are the long-wavelength perturbations. Thus, we focus on the limit in which the wavelength of the perturbations is much larger than the film depth. The energy of a liquid film within this small slope approximation is

$$
\begin{aligned}
\mathcal{E}= & \int d x \int d y\left\{\left(\frac{\gamma}{2} \nabla h\right)^{2}+U\left[h(x)-h_{s}(x)\right]\right. \\
& \left.+\int_{0}^{h(x)} d z \rho g z-P \int_{0}^{h(x)} d z\right\},
\end{aligned}
$$


where $U$ is the interaction potential between the substrate and the surface, $g$ is the gravity constant, $\rho$ is the liquid density, and $P$ is a Lagrange multiplier to impose a constant liquid volume. As opposed to the assumptions described above, we assume here that small perturbations of the free surface $h^{(1)}(x)$ and of the substrate $h_{s}^{(1)}(x)$ around their average heights are of the same order of magnitude. We then obtain a quadratic form for the energy

$$
\begin{aligned}
\mathcal{E}= & \int d x \int d y \frac{1}{2}\left\{\gamma\left[\nabla h^{(1)}(x)\right]^{2}\right. \\
& \left.+\left[h^{(1)}(x)-h_{s}^{(1)}(x)\right]^{2} U^{\prime \prime}\left(\bar{h}_{s}\right)+\rho g\left[h^{(1)}(x)\right]^{2}\right\},
\end{aligned}
$$

leading to an equilibrium equation

$$
-\gamma \nabla^{2} h^{(1)}(x)+\left[U^{\prime \prime}\left(\bar{h}_{s}\right)+\rho g\right] h^{(1)}(x)=U^{\prime \prime}\left(\bar{h}_{s}\right) h_{s}^{(1)}(x) .
$$

Consider, for example, a sinusoidal substrate profile $h_{s}^{(1)}(x)=$ $\zeta_{s} \cos q_{s} x$. Then, the static profile of the free surface is $h_{s}^{(1)}(x)=\zeta \cos q_{s} x$, with

$$
\frac{\zeta}{\zeta_{s}}=\frac{U^{\prime \prime}\left(\bar{h}_{s}\right)}{\gamma q_{s}^{2}+U^{\prime \prime}\left(\bar{h}_{s}\right)+\rho g} .
$$

The static average profile $h_{s}^{(1)}(x)$ must be compared to the amplitude of the fluctuations of the free surface. Since we consider small substrate perturbations, thermal fluctuations only probe the average film depth, and they are therefore independent of $h_{s}^{(1)}(x)$ to leading order. From equipartition, one finds the static spectrum

$$
\left\langle\left|h_{q}\right|^{2}\right\rangle_{\text {stat }}=\frac{k_{B} T}{\gamma q^{2}+U^{\prime \prime}\left(\bar{h}_{s}\right)+\rho g},
$$

leading to a surface roughness

$$
W_{\mathrm{th}}=\left[\frac{k_{B} T}{2 \gamma} \ln \left(1+\frac{\gamma q_{c}^{2}}{U^{\prime \prime}\left(\bar{h}_{s}\right)+\rho g}\right)\right]^{1 / 2},
$$

where $q_{c}$ is an ultraviolet cutoff coming from the discrete nature of the interface, and is usually taken to be equal to a molecular length. Comparison with Eq. (20) indicates that $\Pi^{\prime}\left(\bar{h}_{s}\right)=U^{\prime \prime}\left(\bar{h}_{s}\right)+\rho g$.

To gain quantitative insights into the comparison between the average deformation of the free interface and thermal fluctuations, we consider van der Waals interactions, leading to [20]

$$
U(z)=\frac{A}{12 \pi z^{2}}
$$

with the Hamaker constant $A$. Usually, $|A| \approx 10^{-20} \mathrm{~J}$. When $A>0$, the film wets the substrate and is stable for any thickness. However, when $A<0$, there is a critical thickness

$$
h_{\text {spinod }}=\left(\frac{2 \pi \rho g}{-A}\right)^{1 / 4}
$$

below which the film is unstable. Using typical numbers $\rho \approx$ $10^{3} \mathrm{~kg} \mathrm{~m}^{-3}$ and $g \approx 10 \mathrm{~m} \mathrm{~s}^{-2}$, we find $h_{\text {spinod }} \approx 600 \mathrm{~nm}$.

A quantitative comparison between the average deformation of the free interface and the thermal roughness of the interface is given in Fig. 5. The results are shown for $A=10^{-20}$ and $-10^{-20} \mathrm{~J}$ using the surface tension of water,
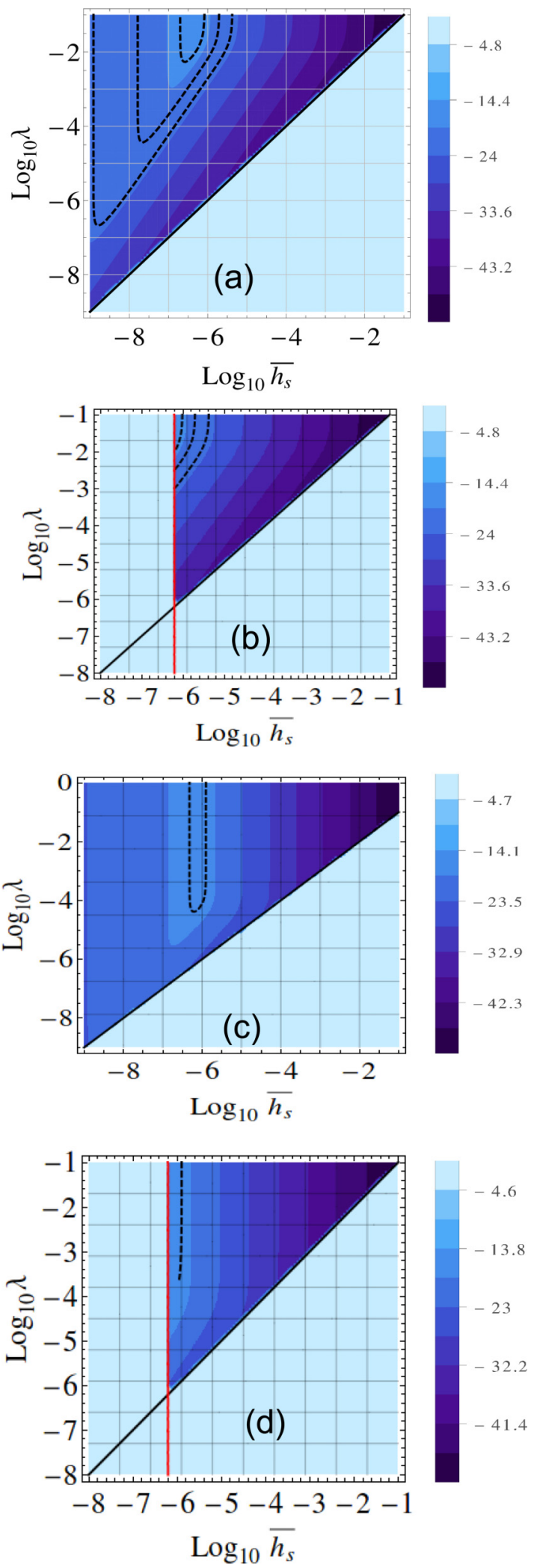

FIG. 5. Amplitude of the static roughness for $\zeta_{s}=0.1 \bar{h}_{s}$, and $|A|=10^{20} \mathrm{~J}$ (all lengths are in $\mathrm{m}$ ). (a,b) Case of water. The dashed lines correspond to $\zeta / 2=0.1 W_{\text {th }}, \zeta / 2=W_{\text {th }}$, and $\zeta / 2=10 W_{\text {th }}$. (c,d) Case of the experiments of Aarts et al. [2]. The dashed lines correspond to a static roughness equal to $\zeta=W_{\text {th }} / 30$ in (a) and $\zeta=W_{\text {th }}$ in (b). Parts $(\mathrm{a}, \mathrm{c})$ and $(\mathrm{b}, \mathrm{d})$, respectively, correspond to positive and negative Hamaker constants. 
$\gamma=7.2 \times 10^{-2} \mathrm{~J} \mathrm{~m}^{-2}$ with $\zeta_{s}=10^{-1} \bar{h}_{s}$. The blue (gray) scale represents the average roughness for $\zeta_{s}=10^{-1} \bar{h}_{s}$. Since we have used the small slope approximation, we only consider the case $\lambda_{s}=\left(2 \pi / q_{s}\right) \gg \bar{h}_{s}$. The dashed curves represent different ratios of the thermal roughness $W_{\text {th }}$ over the average roughness of the free surface $\zeta / 2$. The results show that it is in general safe to assume that the average roughness is negligible as compare to the thermal roughness for films thicker than $5 \mu \mathrm{m}$. However, for thinner films, such as, for example, with $\bar{h}_{s}=100 \mathrm{~nm}$, the substrate wavelength $\lambda_{s}$ should be smaller than $100 \mu \mathrm{m}$ when $A>0$.

These results indicate that the SFSR spectroscopy method, in which films are typically thicker than some micrometers, falls in the range where the average roughness induced by the pattern is negligible.

Similar results for the experiments of Aarts et al. [2], with $\gamma=10^{-7} \mathrm{~J} \mathrm{~m}^{-2}$ and $|A|=10^{-20} \mathrm{~J}$, indicate once again that it is safe to neglect the static roughness for film thicknesses larger than several micrometers.

However, our description, which assumes small fluctuations, fails when fluctuations are so large that the free surface collides with the substrate. Such a substrate-induced confinement of the fluctuations was observed experimentally close to criticality in Ref. [21]. In this situation, surface fluctuations tend to thicken the thin film due to the entropic repulsion between the free surface and the substrate [21]. As a consequence, we expect both the static spectrum (and therefore the roughness) and the average height of the free surface to be influenced by surface patterning in this regime. Since they are a consequence of large fluctuations, these effects cannot be probed by our model, where we assume that surface fluctuations are always much smaller than the film thickness. To address this question, one should, e.g., resort to a renormalization-group approach similar to that of Ref. [22].

\section{APPENDIX B: DERIVATION OF THE SURFACE RELAXATION RATE}

In this Appendix, the main steps of the derivation of the surface relaxation rate are reported. First, using Eqs. (1) and (2), we find the general expression of the velocity and pressure as

$$
\begin{aligned}
u_{z \omega \mathbf{q}}(z)= & C_{1} \cosh q z+C_{2} \sinh q z+C_{3} q z \cosh q z \\
& +C_{4} q z \sinh q z \\
q^{2} u_{x \omega \mathbf{q}}(z)= & i q_{x} \partial_{z} u_{z \omega \mathbf{q}}(z)+i q_{y}\left(D_{1} \cosh q z+D_{2} \sinh q z\right),
\end{aligned}
$$

$$
q^{2} u_{y \omega \mathbf{q}}(z)=i q_{y} \partial_{z} u_{z \omega \mathbf{q}}(z)-i q_{x}\left(D_{1} \cosh q z+D_{2} \sinh q z\right)
$$

$$
q^{2} p_{\omega \mathbf{q}}(z)=\eta\left(\partial_{z z}-q^{2}\right) \partial_{z} u_{z \omega \mathbf{q}},
$$

where $\mathbf{q}=\left(q_{x}, q_{y}\right)$ and $q=|\mathbf{q}|$. The six parameters $C_{i}, D_{i}$ determine completely the velocity and the pressure.

From the condition Eq. (5) at the free surface to leading order in $\delta h$, we may now rewrite the velocities as a function of three parameters,

$$
\begin{aligned}
u_{z \omega \mathbf{q}}(z)= & C_{1}(\cosh q z-q z \sinh q z) \\
& -\frac{\epsilon_{q}}{2 \eta q} \delta h_{q} \sinh q z+C_{3} q z \cosh q z, \\
u_{x \omega \mathbf{q}}(z)= & q^{-2}\left[i q_{x} \partial_{z} u_{z \mathbf{q}}(z)+i q_{y} D_{1} \cosh q z\right], \\
u_{y \omega \mathbf{q}}(z)= & q^{-2}\left[i q_{y} \partial_{z} u_{z \mathbf{q}}(z)-i q_{x} D_{1} \cosh q z\right] .
\end{aligned}
$$

To linear order in $\delta h_{s}$, the substrate boundary condition (3) reads

$$
\mathbf{0}=\mathbf{u}\left(x, y,-\bar{h}_{s}, t\right)+\delta h_{s}(x, y) \partial_{z} \mathbf{u}\left(x, y,-\bar{h}_{s}, t\right) .
$$

The velocity field $\mathbf{u}$ and the free-surface fluctuation $\delta h$ are expanded to leading order in $\delta h_{s}$. In Fourier space, we have

$$
\begin{gathered}
\mathbf{u}_{\omega \mathbf{q}}(z)=u_{\omega \mathbf{q}}^{(0)}(z)+u_{\omega \mathbf{q}}^{(1)}(z)+\cdots, \\
\delta h_{\omega \mathbf{q}}=\delta h_{\omega \mathbf{q}}^{(0)}+\delta h_{\omega \mathbf{q}}^{(1)}+\cdots .
\end{gathered}
$$

Upon substitution in Eq. (B8), we find to zeroth order (i.e., for a flat substrate)

$$
\mathbf{u}_{\omega \mathbf{q}}^{(0)}\left(-\bar{h}_{s}\right)=0
$$

This relation allows one to obtain the velocity field $\mathbf{u}_{\omega \mathbf{q}}^{(0)}$. To first order, Eq. (B8) leads to

$$
\mathbf{u}_{\omega \mathbf{q}}^{(1)}\left(-\bar{h}_{s}\right)+\int \frac{d \mathbf{q}^{\prime}}{(2 \pi)^{2}} \delta h_{s \mathbf{q}-\mathbf{q}^{\prime}} \partial_{z} \mathbf{u}_{\omega \mathbf{q}^{\prime}}^{(0)}\left(-\bar{h}_{s}\right)=0 .
$$

Using this relation, one obtains the expression of the velocity field to first order $\mathbf{u}_{\omega \mathbf{q}}^{(1)}$.

Finally, to linear order in $\delta h$, the interface kinematic equation (7) reads

$$
\partial_{t} \delta h(x, y, t)=u_{z}(x, y, 0, t)
$$

Since the expression of the velocities $\mathbf{u}$ is now known up to first order in $\delta h_{s}$, Eq. (B13) provides the dispersion relation given in the main text, Eq. (11).

\section{APPENDIX C: SPECIFIC SUBSTRATE GEOMETRIES}

\section{Sinusoidal parallel grooves}

We would like to determine the fluctuation spectrum resulting from a sinusoidal profile along the $x$ axis:

$$
\delta h_{s}(\mathbf{r})=\delta \bar{h}_{s} \cos \left(\mathbf{q}_{0} \cdot \mathbf{r}\right)=\delta \overline{h_{s}} \cos \left(q_{0} x\right),
$$

where $\bar{\delta} \bar{h}_{s}$ is a constant, which leads to

$$
\delta h_{s \mathbf{q}}=(2 \pi)^{2} \delta_{q_{y}} \frac{1}{2}\left(\delta_{q_{0 x}-q_{x}}+\delta_{q_{0 x}+q_{x}}\right) \delta \bar{h}_{s} .
$$

Thus,

$$
\mathcal{S}_{\omega}^{(1)}(\mathbf{r})=\frac{\delta h_{s}}{2}\left[e^{i \mathbf{q}_{0} \cdot \mathbf{r}} \mathcal{C}_{\omega \mathbf{q}_{0}}^{-1}+e^{-i \mathbf{q}_{0} \cdot \mathbf{r}} \mathcal{C}_{\omega-\mathbf{q}_{0}}^{-1}\right]
$$

Using the symmetry $\mathcal{C}_{\omega-\mathbf{q}_{0}}^{-1}=\mathcal{C}_{\omega \mathbf{q}_{0}}^{-1}$, we find

$$
\mathcal{S}_{\omega}^{(1)}(\mathbf{r})=\delta h_{s}(\mathbf{r}) \mathcal{C}_{\omega \mathbf{q}_{0}}^{-1} .
$$




\section{Square parallel grooves}

The Fourier cosine representation of a square pattern of height $2 \bar{\delta} h_{s}$ and periodicity $\lambda$ is

$$
h_{s}(\mathbf{r})=\frac{4 \delta \bar{h}_{s}}{\pi} \sum_{n=0}^{\infty} \frac{(-1)^{n}}{2 n+1} \cos \left[(2 n+1) \frac{2 \pi x}{\lambda}\right] .
$$

Hence, the space-dependent part of the power spectrum in the presence of a substrate with parallel grooves with a square cross section is

$$
\mathcal{S}_{\omega}^{(1)}(\mathbf{r})=\frac{4 \delta \bar{h}}{\pi} \sum_{n=0}^{\infty} \frac{(-1)^{n}}{2 n+1} \mathcal{C}_{\omega, \hat{x}(2 n+1) 2 \pi / \lambda}^{-1} .
$$

\section{Localized Gaussian hole or protrusion}

Let us assume that the substrate exhibits an isolated protrusion or hole, with

$$
\delta h_{s}(\mathbf{r})=\delta \bar{h}_{s} e^{-r^{2} / a^{2}}
$$

Then

$$
h_{\mathbf{q}}=\pi a^{2} \bar{\delta} \bar{h}_{s} e^{-q^{2} a^{2} / 4},
$$

and from Eq. (42) we have

$$
\mathcal{S}_{\omega}^{(1)}(\mathbf{r})=\pi a^{2} \delta \bar{h}_{s} \int \frac{d \mathbf{q}}{(2 \pi)^{2}} e^{i \mathbf{q} \cdot \mathbf{r}-q^{2} a^{2} / 4} \mathcal{C}_{\omega \mathbf{q}}^{-1}
$$

[1] T. M. Haruo Sato and M. C. Fehler, Seismic Wave Propagation and Scattering in the Heterogeneous Earth: Second Edition (Springer, Berlin, 2012).

[2] D. G. A. L. Aarts, M. Schmidt, and H. N. W. Lekkerkerker, Science 304, 847 (2004).

[3] A. Tay, C. Thibierge, D. Fournier, C. Fretigny, F. Lequeux, C. Monteux, J. P. Roger, and L. Talini, Rev. Sci. Instrum. 79 103107 (2008)

[4] D. M. Huang, C. Sendner, D. Horinek, R. R. Netz, and L. Bocquet, Phys. Rev. Lett. 101, 226101 (2008).

[5] P. Joseph, C. Cottin-Bizonne, J. M. Benoît, C. Ybert, C. Journet, P. Tabeling, and L. Bocquet, Phys. Rev. Lett. 97, 156104 (2006).

[6] B. Pottier, E. Verneuil, L. Talini, and O. Pierre-Louis, Phys. Rev. E 89, 052403 (2014).

[7] L. Landau and E. Lifchitz, Theoretical Physics 6: Hydrodynamics (Butterworth-Heinemann, Oxford, 1999).

[8] M. L. Henle and A. J. Levine, Phys. Rev. E 75, 021604 (2007).

[9] T. Bickel (unpublished).

[10] G. Grün, K. Mecke, and M. Rauscher, J. Stat. Phys. 122, 1261 (2006).

[11] M. Thiébaud and T. Bickel, Phys. Rev. E 81, 031602 (2010).

[12] A. B. D. Cassie and S. Baxter, Trans. Faraday Soc. 40, 546 (1944).
[13] D. Quéré, Rep. Prog. Phys. 68, 2495 (2005)

[14] R. J. Vrancken, H. Kusumaatmaja, K. Hermans, A. M. Prenen, O. Pierre-Louis, C. W. M. Bastiaansen, and D. J. Broer, Langmuir 26, 3335 (2010).

[15] K. J. Alvine, Y. Dai, H. W. Ro, S. Narayanan, A. R. Sandy, C. L. Soles, and O. G. Shpyrko, Phys. Rev. Lett. 109, 207801 (2012).

[16] A. Mamane, C. Frétigny, F. Lequeux, and L. Talini, Europhys. Lett. 88, 58002 (2009).

[17] A. Cuenca and H. Bodiguel, Phys. Rev. Lett. 110, 108304 (2013).

[18] J. R. Dorgan and N. A. Rorrer, Phys. Rev. Lett. 110, 176001 (2013).

[19] P.-G. de Gennes, F. Brochard-Wyart, and D. Qéré, Capillarity and Wetting Phenomena: Drops, Bubbles, Pearls, Waves (Springer, New York, 2003).

[20] J. N. Israelachvili, Intermolecular and Surface Forces (Elsevier, Amsterdam, 2011).

[21] Y. Hennequin, D. G. A. L. Aarts, J. O. Indekeu, H. N. W. Lekkerkerker, and D. Bonn, Phys. Rev. Lett. 100, 178305 (2008).

[22] A. O. Parry, J. M. Romero-Enrique, and A. Lazarides, Phys. Rev. Lett. 93, 086104 (2004). 